\title{
LncRNA SNHG16 Functions as an Oncogene by Sponging MiR-4518 and Up- Regulating PRMT5 Expression in Glioma
}

\author{
Ye-Fen Lu ${ }^{a} \quad$ Xue-Li Cai ${ }^{a} \quad$ Zheng-Zheng Lib Jing Lv ${ }^{c} \quad$ Yi-an Xiang ${ }^{a} \quad J i a n-J u n ~ C h e n^{a}$ \\ Wei-Jing Chen ${ }^{a} \quad$ Wei-Yan Sun $^{a} \quad$ Xiu-Mei Liu ${ }^{a} \quad$ Jian-Bo Chen ${ }^{c}$ \\ aDepartment of Neurology, The Fifth Affiliated Hospital of Wenzhou Medical University, Lishui, \\ Zhejiang, 'Department of Neurology, The First Affiliated Hospital of Wenzhou Medical University, \\ Wenzhou, Zhejiang, 'Department of Otorhinolaryngology, The sixth Affiliated Hospital of Wenzhou \\ Medical University, Lishui, Zhejiang, China
}

\section{Key Words}

Glioma • Long non-coding RNA • SNHG16 • MiR-4518 • PRMT5

\begin{abstract}
Background/Aims: Long noncoding RNAs (IncRNAs) have recently emerged as novel and potentially promising therapeutic targets in various cancers. However, the expression pattern and biological function of IncRNAs in glioma remain largely elusive. In the present study, we investigated the functional role of an IncRNA, small nucleolar RNA host gene 16 (SNHG16), in glioma. Methods: The expression levels of SNHG16 and miR-4518 were measured using qRT-PCR. The relationship between the levels of SNHG16 and clinicopathologic features were statically analyzed. The levels of proteins were detected using western blot. Bioinformatics analysis and luciferase reporter assays were applied to the analysis of the relationship between SNHG16, miR-4518 and PRMT5. Cell viability and apoptosis were measured using MTT and apoptosis ELISA assay, respectively. Results: SNHG16 was highly expressed in glioma tissues and cell lines, which was related to poorer clinicopathologic features and shorter survival time. Knockdown of SNHG16 inhibits the viability and induces apoptosis of glioma cells. Further investigation revealed that SNHG16 could up-regulate the expression of miR-4518 targeted gene PRMT5 via acting as an endogenous sponge of miR-4518. Moreover, SNHG16 also affects the expression of $\mathrm{Bcl}-2$ family proteins and the activation of PI3K/Akt signaling pathway. Conclusion: Our study revealed a novel SNHG16-miR-4518-PRMT5 pathway regulatory axis in glioma pathogenesis. SNHG16 could be used as a potential therapeutic target in the treatment of glioma.

\section{Introduction}

Glioma is the most common and aggressive tumor in the central nervous system, accounting for $70 \%$ of adult malignant primary brain tumors [1]. Despite the diagnosis Y.-F. Lu and X.-L. Cai contributed equally to this work.

Jian-Bo Chen

KARGER
Department of Otorhinolaryngology,

The sixth Affiliated Hospital of Wenzhou Medical University, Lishui, Zhejiang, (China) E-Mail chenjianbo.516@163.com 
and treatment strategies that have been developed in the past decade, gliomas are still incurable, and the prognosis of these patients still remains poor, especially for patients with glioblastoma multiforme (GBM) [2]. The median survival time of glioma patients is normally within twelve months after diagnosis, and most patients will live no longer than two years, even in the most advantageous situations [3]. Therefore, it is urgent to unveil the underlying mechanisms in glioma tumorigenesis and to develop more effective therapeutic strategies.

Long non-coding RNAs (IncRNAs) are a set of RNAs with size over 200 nucleotides that do not have an open read frame (ORF) but can regulate gene expression at pre-transcriptional, post-transcriptional and epigenetic levels [4]. LncRNA plays an important role in various vital biological process such as differentiation, metabolism, migration and apoptosis [5]. In recent years, several lncRNAs have been identified as being involved in the development of glioma. For example, IncRNA HOTAIR has been found to be associated with the survival of glioma patients and thus can be applied as a prognostic factor [6]. LncRNA MALAT1 promoted the proliferation and inhibited apoptosis, thus exhibiting oncogenic function in glioma cells [7]. Moreover, MALAT1 could also affect glioma cells response to temozolomide [8]. Although many lncRNAs have been identified to exert regulating activities during the development and progression of glioma, the role of the vast majority of these transcripts is still elusive.

Small nucleolar RNA host gene 16 (SNHG16), a newly identified IncRNA, is also known to be a noncoding RNA (ncRAN) that is expressed in aggressive neuroblastoma. Increased expression of SNHG16 has been found to be associated with poor outcomes in neuroblastoma patients [9]. Overexpression of SNHG16 promotes the invasiveness of bladder cancer [10]. In contrast, reduced SNHG16 expression was found to be associated with poor prognosis in colorectal cancer [11]. However, there is little knowledge about the role of SNHG16 in the progression of glioma.

In the present study, we found that SNHG16 was up-regulated in glioma tissues and cell lines, and its high-expression was associated with tumor grade and poor prognosis in glioma patients. Furthermore, in vitro experiments were applied to investigate the functions of SNHG16 in glioma tumorigenesis. Our study indicated that SNHG16 acts as an oncogene via sponging miR-4518 and up-regulating PRMT5 expression in glioma. Therefore, SNHG16 may be applied as a potential target in glioma therapy.

\section{Materials and Methods}

\section{Clinical tissue specimens}

Forty-eight paired brain glioma specimens and the peritumoral brain edema (PTBE) tissues were collected from surgical tumor resections performed in the fifth Affiliated Hospital of Wenzhou Medical University and in the sixth Affiliated Hospital of Wenzhou Medical University. The samples were collected between 2014-2017. The tissues were snap-frozen in liquid nitrogen and stored at $-80^{\circ} \mathrm{C}$ for the subsequent analysis. All the tissues were obtained with informed consent and this study was approved by the research ethics committee at the Wenzhou Medical University.

\section{Cell culture and small interfering RNA (siRNA) for transfection into}

NHAs (normal human astrocytes), U251, H4, SW1783 and LN229 cells were purchased from Shanghai Bank of Tissues, Shanghai, China. All the cells were maintained in Dulbecco's Modified Eagle's Medium (Gibco BRL, Grand Island, NY, USA) containing 10\% fetal bovine serum (FBS) (Gibco BRL) and maintained at $37^{\circ} \mathrm{C}$ with $5 \% \mathrm{CO} 2$. The glioma cell lines were transfected with siRNA using Lipofectamine 2000 (Invitrogen, Carlsbad, CA, USA) according to the manufacturer's guide. The siRNAs (si-NC, siSNHG16, si-PRMT5), miRNA mimics (miR-NC, miR-4538), anti-miR-4518 and overexpression plasmids (pcDNA, pcDNA-SNHG16) were synthesized by Shanghai GenePharma Co., Ltd. (Shanghai, China). The full length of SNHG16 was amplified from human cDNA by PCR (F: CCCAAGCTTGCGTTCTTTTCGAGGTCGGC; R: CCGGAATTCTGACGGTAGTTTCCCAAGTTTATTGTAAGT) and inserted into pcDNA3.1 vector (Invitrogen, USA). The target sequences of siRNA were as follows: si-NC, UUCUCCGAACGUGUCACGUTT; si-SNHG16, GGAAUGAAGCAACUGAGAUUU; si-PRMT5 GCAGACGCUCUGGUUGUUA. 


\section{RNA isolation and quantitative PCR}

Total RNA was isolated using the TRIZOL Reagent (Invitrogen, USA) according to the manufacturer's guide. cDNA was synthesized from $1 \mu \mathrm{g}$ of RNA in a final volume of $20 \mu \mathrm{L}$ with random primers using the High Capacity cDNA Reverse Transcription Kit (Applied Biosystems, USA). The Fast Start Universal SYBR Green Master (Roche, USA) was applied for the quantitative RT-PCR. The primers were obtained from GenePharma. The sequences were shown as follows: SNHG16 F: GCAGAATGCCATGGTTTCCC; R: GGACAGCTGGCAAGAGACTT; miR-4518 F: GGAGGGATGATAACTGTGCTGAGA; R: TCAATCCCA

GCTCTTTT; PRMT5 F: CTGACACACTAGGGGCTGTG; R:5'-ACTAGTCTGCCCTTCTCCGT; GAPDH F: AGAAGGCTGGGGCTCA

TTTG, R: AGGGGCCATCCACAGTCTTC. The 2- $\Delta \Delta \mathrm{Ct}$ method was used to analyze the relative fold changes, where $\mathrm{Ct}$ is cycle thresholds.

\section{Cell viability assay}

Cell viability was assayed using the Cell Counting Kit-8 (CCK8; Beyotime, Beijing) according to the manufacturer's guide. Briefly, cells $(2,000$ cells/well) in each group were plated into 96 -well plates. The CCK8 regent $(\sim 10 \mu \mathrm{L})$ was added to each well containing $100 \mu \mathrm{L}$ medium after transfection and then incubated for 2 hours at $37^{\circ} \mathrm{C}$ with $5 \% \mathrm{CO} 2$. The plates were measured at a wavelength of $450 \mathrm{~nm}$ using a microplate reader. Five replicate wells were designed in each group and experiments were repeated in triplicate.

\section{Western blot analysis}

Cells were collected and lysed with RIPA lysis buffer (Beyotime, Beijing, China) with complete Protease Inhibitor Cocktail (Roche, USA). Equal amounts of protein lysates were subjected to 12\% SDS-PAGE gels and then transferred to PVDF membrane (Millipore, Billerica, MA, USA). The membranes were incubated with the primary antibodies at $4^{\circ} \mathrm{C}$ overnight. The following primary antibodies were used: Caspase-3 (Cell Signaling, USA), Cleaved PARP (Cell Signaling, USA), Bcl-2 (Cell Signaling, USA), Bcl-xl (Cell Signaling, USA), Mcl-1(Cell Signaling, USA), Bax (Cell Signaling, USA), AKT (Cell Signaling, USA), phospho-AKT (Cell Signaling, USA), PI3K (Cell Signaling, USA), phospho-PI3K (Cell Signaling, USA), PRMT5 (Abcam, USA), and GAPDH (Cell Signaling, USA). After incubating with secondary horseradish peroxidase-conjugated antibodies (Cell Signaling, USA) for $2 \mathrm{~h}$ at room temperature, blots were visualized through an enhanced chemiluminescence system (Thermo Fisher Scientific, USA). Protein bands were quantified by densitometric analysis using Quantity One software (Bio-Rad Laboratories, San Diego, CA, USA).

\section{Caspase-3 activity assay}

Caspase-3 activity was assayed using the caspase-3 activation kit (R \& D Systems, Minneapolis, MN, USA) according to the manufacturer's instructions. Briefly, cell lysates were prepared and incubated with the supplied reaction buffer and the colorimetric substrates (Ac-DEVD-AMC) at $37^{\circ} \mathrm{C}$ for $1 \mathrm{~h}$ in the dark. Then, the absorbance of the solutions was measured on a BioTek microplate reader at $405 \mathrm{~nm}$.

\section{Apoptosis assay}

For apoptosis assays, cells were harvested after different treatments and the apoptosis rate was measured using the Nucleosome ELISA Kit (Millipore, Darmstadt, Germany) according to the manufacturer's instruction.

\section{Luciferase reporter assays}

SNHG16 fragment containing the predicated miR-4518 binding site, the wide-type or mutant putative sequences of the binding site were subcloned into a pmirGLO Dual-luciferase vector (Promega, USA) to form the reporter vector pmiRGLO-SNHG16-wild type (SNHG16-wt) or pmiRGLO-SNHG16-mutant (SNHG16mut). SNHG16-wt or SNHG16-mut were co-transfected with miR-4518 mimics or negative control (miRNC) into glioma cells by using Lipofectamine 2000. $48 \mathrm{~h}$ after transfection, the relative luciferase activities were measured by a dual-luciferase reporter assay system (Promega, USA) according to the manufacturer's guide. Similarly, the 3'UTR of PRMT5 containing the miR-4518 binding site, the wild-type or mutant putative sequences of the binding site were cloned into a pmirGLO Dual-luciferase vector to form the reporter vector pmiRGLO-PRMT5-wild-type (PRMT5-wt) or pmiRGLO-PRMT5-mutant (PRMT5-mut). The resulting 
constructs were co-transfected with miR-4518 mimics, miR-NC mimics into cells using Lipofectamine 2000 according to the manufacturer's guide. At $48 \mathrm{~h}$ after transfection, the relative luciferase activities were measured.

\section{Statistical analysis}

All data are presented as the mean \pm standard deviation (SD) from three independent experiments. All statistical analyses were performed by using SPSS 18.0 software (IBM, USA). Differences between groups were analyzed by using Student's t-test (two groups) or one-way ANOVA analysis (multiple groups). A chisquare test was applied to determine the association of SNHG16 levels with clinicopathological features. Overall survival (OS) was defined as the time from surgery to death or to the date of the last recorded followup visit. A Kaplan-Meier curve was plotted for survival analysis, and the difference between the two groups was compared using a log-rank test. $\mathrm{P}<0.05$ was considered statistically significant.

\section{Results}

SNHG16 is highly expressed in glioma cells and tissues

The expression of SNHG16 was first evaluated in glioma tissues by qRT-PCR. In 48 paired glioma tissues and the PTBE tissues, the expression of SNHG16 was significantly increased in glioma tissues, compared to the PTBE tissues (Fig. 1A). Moreover, the SNHG16 expression in the human glioma cell lines (U251, H4, SW1783 and LN229) was significantly higher than that of NHA cells (all P<0.05), in which LN229 and U251 cells has the highest and the lowest expression, respectively. Thus, those two cell lines were selected to be used in the following studies.

High SNHG16 expression in glioma was related with poorer clinicopathological parameters and shorter overall survival

Forty-eight pairs of glioma tissues were divided into two groups: a low SNHG16 expression level (below the median SNHG16 expression, $\mathrm{n}=23$ ) and a high expression group (above the median SNHG16 expression, $\mathrm{n}=25$ ). As indicated in Table 1, high expression of SNHG16 in glioma tissue was shown to be associated with larger tumor size $(\mathrm{P}=0.009)$ and advanced WHO stage (0.021). To explore the potential relationship between SNHG16 expression and the patients' prognosis, Kaplan-Meier analysis and log-rank test were used to evaluate the effects of SNHG16 expression on overall survival (OS) and progression-free survival (PFS). The results indicated that patients with higher SNHG16 expression had a significantly poorer OS $(\mathrm{P}<0.001)$ and PFS $(\mathrm{P}<0.001)$ (Fig. 2). Then, univariate and multivariate analyses were used to evaluate whether the SNHG16 expression level and various clinicopathological features were independent prognostic parameters of glioma patient outcomes. As shown in Table 2 and Table 3, according to a univariate analysis, WHO grade and SNHG16 expression were statistically significant prognostic factors. Moreover, multivariate analysis indicated that

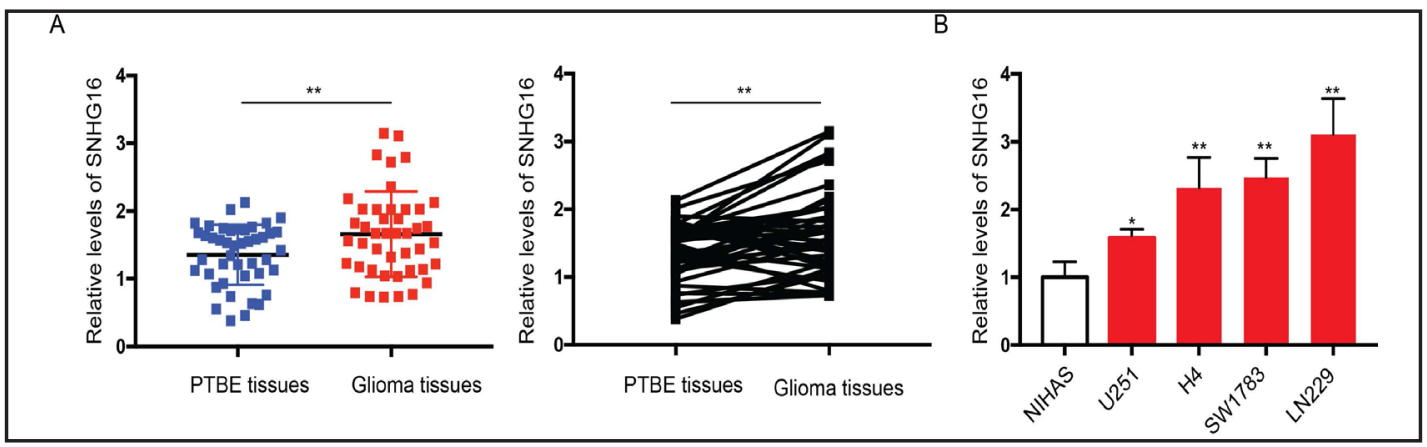

Fig. 1. SNHG16 expression in the human glioma tissues, glioma cell lines and HA cells was evaluated by qRTPCR A, SNHG16 expression in the human glioma and normal tissues was measured by RT-PCR; B, SNHG16 expression in HA, U251, H4, SW1783 and LN229 was measured by RT-PCR. ${ }^{*} \mathrm{P}<0.05,{ }^{* *} \mathrm{P}<0.01$ compared with HA. 
SNHG16 expression was an independent prognostic indicator for the OS $(\mathrm{p}<0.001)$ and PFS $(\mathrm{p}<0.001)$ of patients with glioma.

\section{Down-regulation of SNHG16 inhibited proliferation} and promoted apoptosis in glioma cells

To explore the biological function of SNHG16 in glioma cells, siRNA against SNHG16 were transfected into U251 and LN229 cell lines. Transfection efficiency was confirmed by qRT-PCR analysis (Fig. $3 \mathrm{~A}$ ). CCK-8 assay was performed to determine the effect of SNHG16 on cell proliferation, we found that silencing of SNHG16 significantly inhibited glioma cell proliferation in comparison to negative control (si-NC) (Fig. 3B). Next, an apoptosis ELISA assay was applied to further evaluate whether the effect of SNHG16 on glioma cell proliferation was due to effects on apoptosis. As indicated in Fig. 3C, downregulation of SNHG16 led to increased apoptosis compared with the si-NC group. To further confirm that silencing of SNHG16 resulted in apoptosis in glioma cells, western blot and the caspase- 3 activity assay were carried out to evaluate the activation of caspase- 3 . We found that down-regulation of SNHG16 caused more cleavage and activation of caspase- 3 than the control group (Fig. 3D, E).

SNHG16 affects the expression of Bcl2 proteins and activation of PI3K/Akt pathway

The Bcl-2 family proteins and the PI3K/Akt pathway are vital regulators of apoptosis [12, 13]. Therefore, we asked whether silencing of SNHG16 could affect the expression of Bcl-2 family proteins and/or PI3K/Akt pathway proteins. As shown in Fig. 4A, antiapoptotic $\mathrm{Bcl}-2$ proteins such as Bcl-2, Bcl-xl and Mcl-1 were down-regulated while pro-apoptotic Bcl2 protein Bax was upregulated after the silencing of SNHG16. At the same time, phospho-PI3K and phospho-Akt levels were repressed while the total amount of PI3K and Akt was not affected after the silencing of SNHG16 (Fig. 4B). These results indicate that SNHG16 may affect the
Table 1. Correlation of SNHG16 expression with clinicopathological features in 48 glioma patients. *PTBE: Peritumoral Brain Edema

\begin{tabular}{lccc}
\hline & \multicolumn{2}{c}{ Expression of SNHG16 } & \\
Variable & Low (\%) & High (\%) & P value \\
\hline Gender & & & 0.386 \\
$\quad \begin{array}{l}\text { Female } \\
\text { Male }\end{array}$ & $13(54.2 \%)$ & $11(45.8 \%)$ & \\
Age & $10(41.7 \%)$ & $14(58.3 \%)$ & \\
$\quad<60$ & $9(47.4 \%)$ & $10(52.6 \%)$ & 0.951 \\
$\quad \geq 60$ & $14(48.3 \%)$ & $15(51.7 \%)$ & \\
Tumor size & & & 0.009 \\
$<5 \mathrm{~cm}$ & $16(66.7 \%)$ & $8(33.3 \%)$ & \\
$\geq 5 \mathrm{~cm}$ & $7(29.2 \%)$ & $17(70.8 \%)$ & \\
WHO grade & & & 0.021 \\
I-II & $14(70.0 \%)$ & $6(30.0 \%)$ & \\
III-IV & $11(36.7 \%)$ & $19(63.3 \%)$ & \\
PTBE & & & 0.571 \\
$\quad<1 \mathrm{~cm}$ & $12(52.2 \%)$ & $11(47.8 \%)$ & \\
$\geq 1 \mathrm{~cm}$ & $11(44.0 \%)$ & $14(56.0 \%)$ & \\
\hline
\end{tabular}

Table 2. Univariate and multivariate analyses for progression-free survival by Cox regression model. *PTBE: Peritumoral Brain Edema

\begin{tabular}{lccc}
\hline \multirow{2}{*}{ Parameters } & \multicolumn{3}{c}{ Progression-free survival } \\
& Univariate analysis & Multivariate analysis & \\
& P & HR 95\% CI & P \\
\hline Age & 0.674 & - & - \\
Sex & 0.232 & - & - \\
Tumor Size & 0.189 & - & - \\
PTBE & 0.521 & - & - \\
WH0 grade & 0.024 & $2.037(1.167-5.323)$ & 0.017 \\
SNHG16 expression & 0.0065 & $3.167(1.552-6.231)$ & 0.021 \\
\hline
\end{tabular}

Table 3. Univariate and multivariate analyses for overall survival by Cox regression model. *PTBE: Peritumoral Brain Edema

\begin{tabular}{lccc}
\hline \multirow{2}{*}{ Parameters } & \multicolumn{3}{c}{ Overall survival } \\
& Univariate analysis & Multivariate analysis & \\
P & HR 95\% CI & P \\
\hline Age & 0.218 & - & - \\
Sex & 0.330 & - & - \\
Tumor Size & 0.422 & - & - \\
PTBE & 0.646 & - & - \\
WHO grade & 0.0027 & $2.133(1.223-4.526)$ & 0.0018 \\
SNHG16 expression & $<0.001$ & $1.623(1.489-2.443)$ & $<0.001$ \\
\hline
\end{tabular}

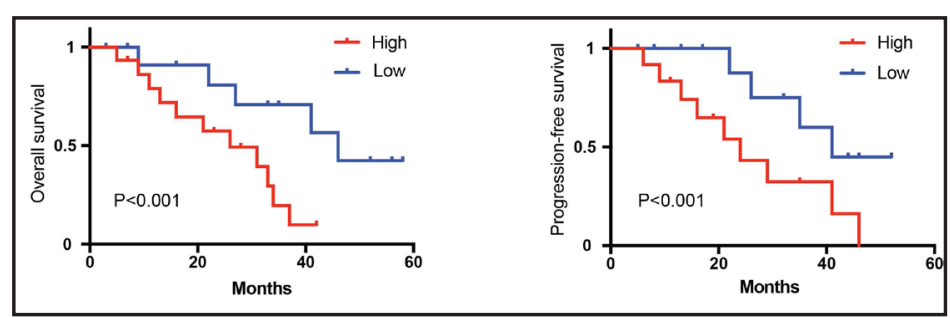

Fig. 2. Up-regulation of SNHG16 was correlated with poor prognosis in glioma patients. A, Kaplan-Meier analysis of the overall survival in glioma patients in relation to SNHG16 expression level. B, KaplanMeier analysis of the progression-free survival in glioma patients in relation to SNHG16 expression level. 
process of apoptosis via regulation of $\mathrm{Bcl}-2$ proteins and inhibition of PI3K/Akt signaling pathway.

SNHG16 directly
interacted with
miR-4518
$\begin{array}{ll}\mathrm{M} \mathrm{o} \mathrm{u} \mathrm{n} \text { i } \mathrm{n} \mathrm{g}\end{array}$
evidence indicated that IncRNAs could function as competing endogenous RNA or a molecular sponge in regulating the biological functions of miRNAs [14]. B i o i n f o r m a t i c s analysis revealed the potential interaction between SNHG16 and miR-4518. There are four binding sites between miR4518 and SNHG16. Each of them is a 7 nucleotides basepair located at the position of 93-99, $1057-1063,1292-$ 1298 and 21932199 at SNHG16 mRNA. Then, luciferase reporter assay was carried out to verify the bio informatical prediction. The results indicated that miR-4518 $\mathrm{s}$ ig n if i c a n t ly repressed the luciferase activity of SNHG16-wt compared to miRNC (negative control), while miR-4518 did not affect the luciferase activity of SNHG-16 mut in both U251 and LN229 cells (Fig. 5A and B). Moreover, we found that overexpression of SNHG16 significantly repressed the expression of miR-4518 and silencing of SNHG16 lead to the up-regulation of miR-4518 in U251 and LN229 cells (Fig. 5C). Further correlation analysis demonstrated that there is a negative correlation between SNHG16 expression and miR-4518 in glioma tissues (R2 $=0.767, \mathrm{P}<0.01$, Fig. 5D). Taken together, these data indicated that miR-4518 could directly bind to SNHG16 in glioma cells.

Fig. 3. SNHG16 promoted proliferation and knockdown of SNHG16 lead to apoptosis in glioma cells. A, The expression of SNHG16 in glioma cell lines transfected with siRNA against SNHG16 (si-SNHG16) or negative control (siNC) were evaluated by RT-PCR. B, CCK-8 assay was used to measure glioma cell proliferation after transfection. C, Apoptosis ELISA assay was used to determine apoptosis in glioma cells after transfection for $24 \mathrm{~h}$. D, After transfection for $24 \mathrm{~h}$, cell lysates were collected and subjected to western blot analysis with indicated antibodies. E, After transfection for $24 \mathrm{~h}$, caspase-3 activity was assayed. ${ }^{* *} \mathrm{P}<0.01,{ }^{* * *} \mathrm{P}<0.001$.

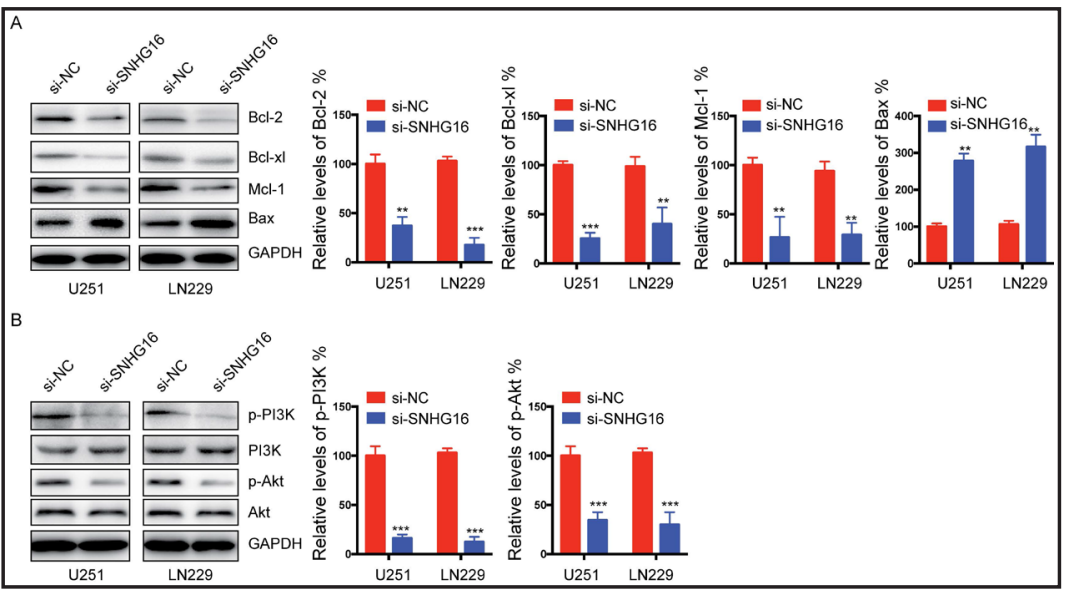

Fig. 4. Down-regulation of SNHG16 affects Bcl-2 family proteins and PI3K/Akt pathway. A and B, after transfection with siRNA against SNHG16 (si-SNHG16) or negative control (si-NC) for $24 \mathrm{~h}$, then total cellular lysates were collected and subjected to the western blot analysis with indicated antibodies. Western blots results were quantitatively analyzed. ${ }^{* *} \mathrm{P}<0.01 ;{ }^{* * *} \mathrm{P}<0.001$.

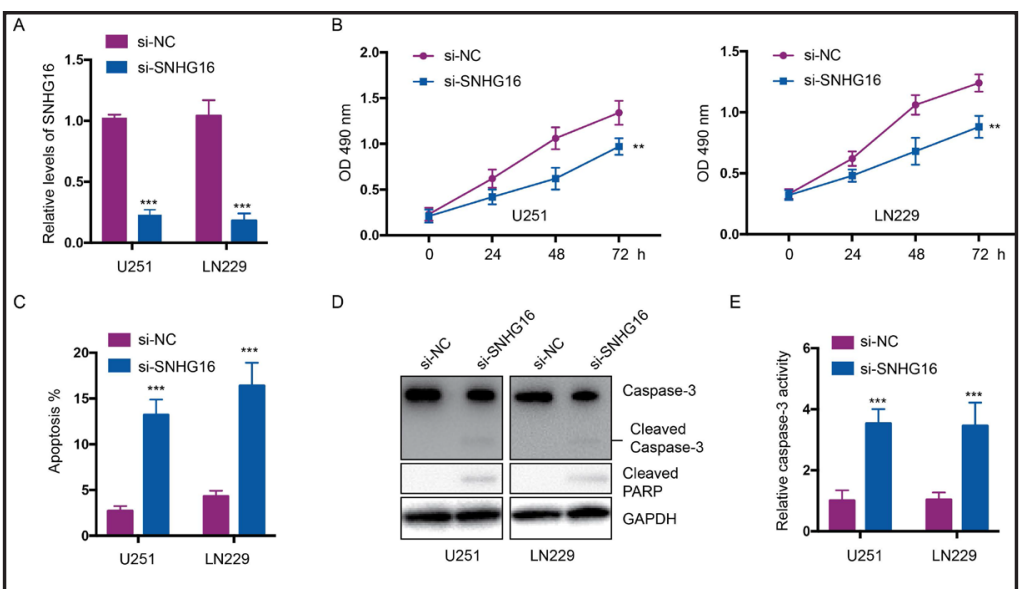




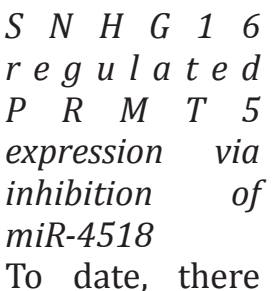
is little knowledge about the function of miR-4518. To further explore the mechanism of SNHG16 in the development of glioma, we $\mathrm{p}$ e $\mathrm{r} f$ o $\mathrm{r}$ m e d b i o informatics analysis and found that PRMT5 is a potential target of miR-4518 (Fig. 6A). Then, we measured the mRNA and protein expression of PRMT5 after transfection with miR-4518 mimics, miR-NC mimics, SNHG16 siRNA and SNHG16 plasmid. We found that PRMT5 mRNA expression was significantly decreased in cells transfected with miR-4518 mimics and SNHG16 siRNA, while it was up-regulated in SNHG16 plasmid transfected glioma cells (Fig. 6B). In line with the RT-PCR results, western blot results showed the same tendency (Fig. 6C, D). Furthermore, luciferase reporter assay showed that overexpression of SNHG16 could restore the luciferase activity of reporter genes carrying PMRT5 3'UTR when compared to empty control in glioma cells (Fig. 6E, F). To further confirm the correlation between PRMT5 and SNHG16, PRMT5 mRNA level was also measured in glioma and PTBE tissues. RT-PCR data showed that PRMT5 expression was significantly up-regulated in glioma tissues compared to PTBE tissues (Fig. 6G). Furthermore, correlation analysis revealed that there is a positive correlation between PRMT5 and SNHG16 (R2=0.534, P<0.01) (Fig. 6H). Taken together, these findings indicated that SNHG16 regulated PRMT5 expression via inhibition of miR-4518 in glioma cells.

\section{Knockdown of PRMT5 induced apoptosis in glioma cells}

To further analyze whether PRMT5 is related to the effect of SNHG16 on glioma cells. First, U251 and LN229 cells were transfected with siRNA against PRMT5. As shown in Fig. 7A and B, the protein and mRNA levels of PRMT5 were significantly down-regulated after transfection with PRMT5 siRNA. Then, we asked whether down-regulation of PRMT5 exerted any effect on the apoptosis of glioma cells. We found that down-regulation of PRMT5 could induce a similar apoptosis rate as silencing of SNHG16 in glioma cells (Fig. 7C). It is noteworthy that inhibition of miR-4518 could decrease the apoptosis induced by silencing of SNHG16 (Fig. 7C). Meanwhile, caspase-3 activity and western blot assay results were in line with the apoptosis assay results (Fig. 7D, E). Moreover, we also investigated the effect of down-regulation of PRMT5 on Bcl-2 family proteins and PI3K/Akt pathway. Like the down-regulation of SNHG16, we found that silencing of PRMT5 showed similar effects on Bcl-2 proteins and PI3K/Akt pathway (Fig. 7F). Taken together, these findings suggested that SNHG16 exerts oncogenic function via the SNHG16-miR-4518-SNHG16 axis in glioma cells. 
Fig. 6. SNHG16 up-regulated PRMT5 via repressing miR-4518 in glioma cells. A, Bioinformatical predication of interaction between miR-4518 and PRMT5. B, Glioma cells were transfected with miR-NC mimics, miR-4518 mimics, SNHG16 plasmid or SNHG16 siRNA, mRNA levels of PRMT5 were detected by RT-PCR. C and D, Glioma cells were transfected with miR-NC mimics, miR-4518 mimics, SNHG16 plasmid or SNHG16 siRNA, protein levels of PRMT5 were detected by western blot. $\mathrm{E}$ and $\mathrm{F}$, The relative luciferase activity of PMRT5 3'UTR in glioma cells transfected with miR-NC mimics, miR-4518 mimics, miR-4518 mimics plus SNHG16 plasmid or miR-4518 mimics plus SNHG16 siRNA. G, PRMT5 mRNA was measured in glioma and PTBE tissues by RT-PCR. H, The correlation between PRMT5 mRNA and SNHG16 expression in glioma tissues. ${ }^{*} \mathrm{P}<0.05$; ${ }^{*} \mathrm{P}<0.01$.

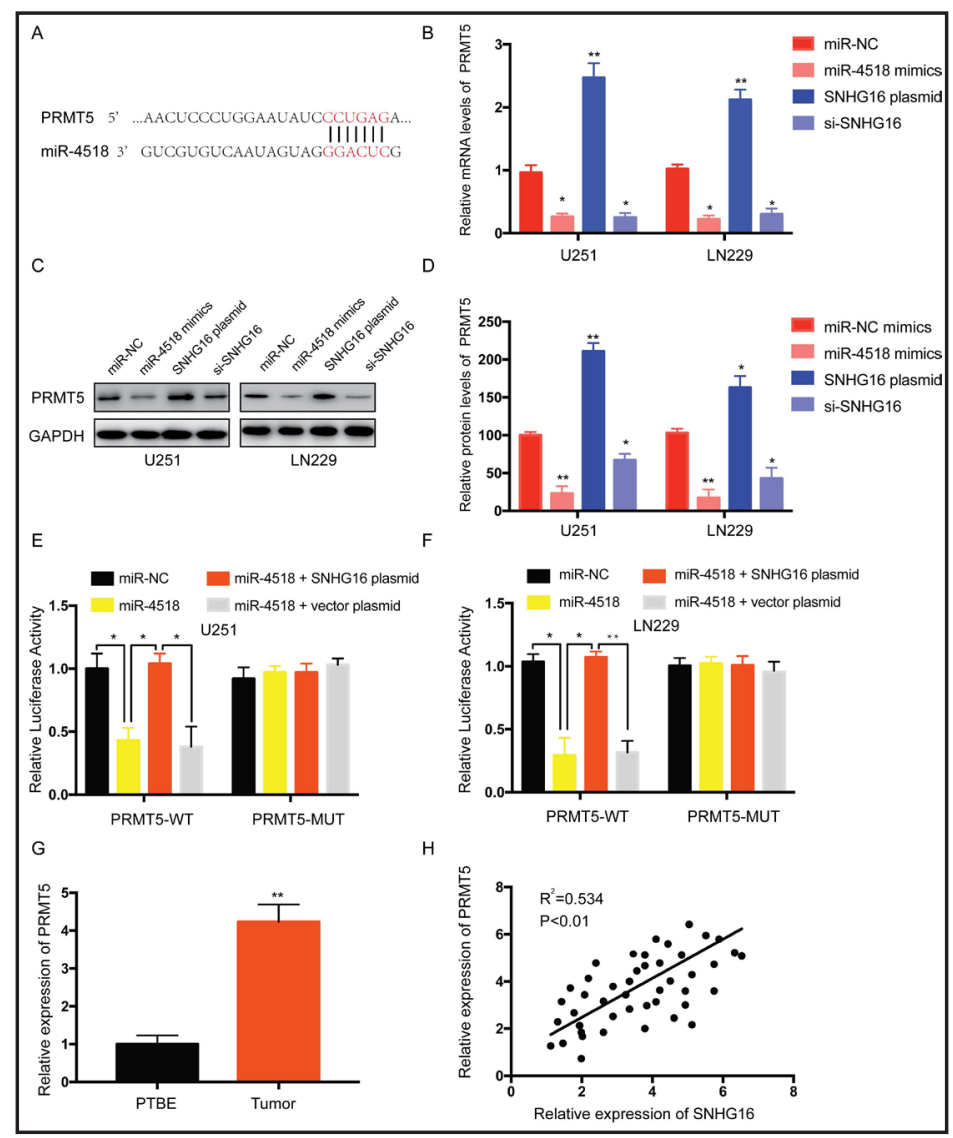

Fig. 7. Knockdown of PRMT5 induced apoptosis on glioma cells. A, After transfection with SiRNA against PRMT5 for $24 \mathrm{~h}$, PRMT5 protein were detected by western blot in glioma cells. B, After transfection with siRNA against PRMT5 for $24 \mathrm{~h}$, mRNA levels of PRMT5 were measured by RT-PCR in gli-
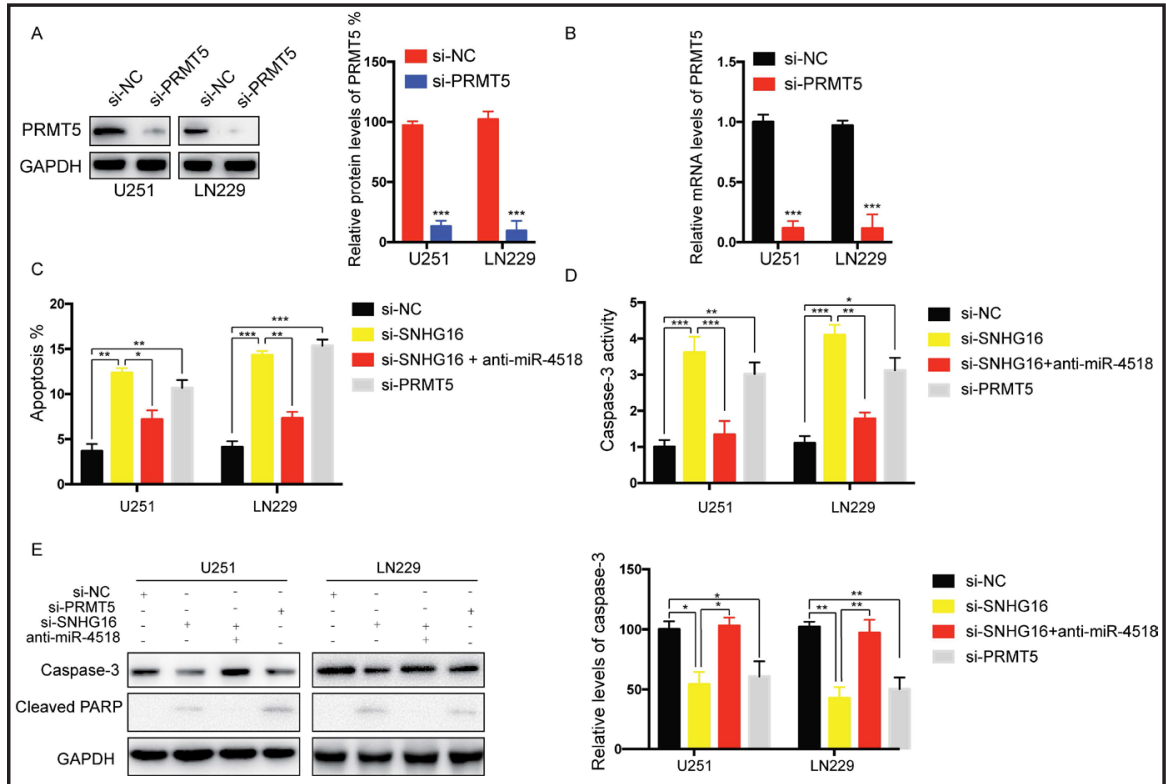
oma cells. C, After

transfection as indicated for $24 \mathrm{~h}$, cellular apoptosis of were detected in glioma cells. D, After transfection as indicated for $24 \mathrm{~h}$, caspase-3 activity was assayed in glioma cells. E and F, After transfection as indicated for $24 \mathrm{~h}$, total cellular lysates were collected and subjected to western blot analysis with indicated antibodies. $* \mathrm{P}<0.05 ;{ }^{* *} \mathrm{P}<0.01 ;{ }^{* * *} \mathrm{P}<0.001$. 


\section{Discussion}

Gliomas account for approximately $30 \%$ of all brain and central nervous system tumors and $80 \%$ of all brain carcinomas [1]. It is very important to identify biomarkers to improve the prognostic outcome of glioma patients. Recently, the emerging role of ncRNAs in glioma tumorigenesis is attracting more and more attention. In the present study, we observed that SNHG16 is involved in the oncogenesis of human glioma cells. Then, bioinformatics analysis indicated the complementary binding sites between SNHG16 and miR-4518. We also found that the expression of miR-4518 was significantly down-regulated in glioma tissues, and correlation analysis revealed the negative correlation between SNHG16 and miR-4518. Moreover, luciferase reporter assay confirmed that miR-4518 might be a target of SNHG16 in glioma cells. SNHG16 also exhibited positive regulation on PRMT5, which has been identified as a direct target of miR-4518. Silencing of PRMT5 showed similar effects on glioma cells as knockdown of SNHG16. In summary, our findings indicated SNHG16 exerted oncogenic functions and involved in the up-regulation of PRMT5 in glioma cells via inhibition of miR4518.

SNHG16 as an oncogenic IncRNA has been reported to be involved in the development of various cancers by different mechanisms of action. For example, SNHG16 has been found to be up-regulated in colorectal cancers and affected the genes involved in lipid metabolism [15]. In a recent study, SNHG16 has been found to be overexpressed and contributed to the development of non-small cell lung cancer (NSCLC) via inhibition of miR-146a [16]. However, there is little knowledge about the role of SNHG16 in the development of glioma. In the present study, we evaluated the expression of SNHG16 and its relationship with the clinical features in the glioma patient. SNHG16 was significantly up-regulated in glioma tissues and cell lines, compared with PTBE and NHA cell lines, respectively. In addition, a higher SNHG16 expression was correlated with a poorer prognosis in glioma patients, including larger tumor size, advanced WHO stages, shorter OS and PFS. To investigate the biological functions of SNHG16 in glioma cells, the loss-of-function assay was performed by SNHG16 knockdown. Knockdown of SNHG16 could repress proliferation and apoptosis in glioma cells. Our findings are consistent with a previous study in which knockdown of SNHG16 could inhibit the proliferation of lung cancer cells [16]. Notably, we also observed that down-regulation of SNHG16 could lead to the repression of anti-apoptotic Bcl-2 proteins and PI3K/Akt pathway. Therefore, targeting Bcl-2 proteins and/or PI3K/Akt pathway could be a promising strategy to fight against gliomas [17-19]. Our findings indicated that SNHG16 might be a potential therapeutic target in treatment of gliomas.

One of the major functions of lncRNAs is to harbor miRNAs to act as a "sponge" and reduce the expression and activity of miRNAs. Therefore, we performed the bioinformatics analysis to identify the miRNAs that can bind to complementary sequences in SNHG16. We found that miR-4518 share the complementary binding sites at 3'-UTR, which was confirmed by the luciferase assay. Loss-of-function revealed that miR-4518 inhibitor could reduce the apoptosis of glioma cells induced by silencing SNHG16. Moreover, the effects of downregulation of SNHG16 on Bcl-2 proteins and PI3K/Akt pathway could be reversed by miR4518 inhibitor as well. Therefore, it might be explained that SNHG16 exerts physiological functions via sponging miR-4518. To date, there is little information about the role of miR4518. Our findings hint that miR-4518 may participate in the tumorigenesis of gliomas and further investigation is required.

LncRNAs can also regulate the expression and biological functions of miRNA targets. Among all of the predicted target genes of miR-4518, we found that PRMT5 played an important role in the development of glioma. Protein arginine methyltransferase 5 (PRMT5) is a member of the PMRT family which covalently modifies histone and non-histone proteins to regulate gene transcription and cellular signaling [20]. Several studies have found that PRMT5 is correlated with the progression of gliomas. For example, PRMT5 has been found overexpressed in glioma cells and associated with more aggressive disease in patients with glioblastoma [21]. In the same study, knockdown of PRMT5 has also been found lead to the 
down-regulation of Bcl-2 and up-regulation of Bax [21]. In a recent study, PRMT5 has been found played a very significant role in glioblastoma neurospheres (GBMNS) self-renewal capacity and proliferation [22]. PRMT5 knockdown also lead to apoptosis and cell cycle arrest in glioma cells [22]. Moreover, the expression of PRMT5 is correlated with malignant grade in gliomas and plays a pivotal role in the proliferation of glioma cells in vitro [23]. These findings are in line with our results that also revealed a novel mechanism that regulated the expression of PRMT5.

\section{Conclusion}

In summary, an SNHG16-miR-4518-PMRT5 regulatory axis in glioma pathogenesis was identified in the present study. SNHG16 acts as an oncogenic lncRNA that promoted glioma tumorigenesis via acting as a competing endogenous RNA that regulated the expression of PRMT5 through directly sponging miR-4518. Our study revealed that SNHG16 could be a potential therapeutic target in the management of glioma.

\section{Disclosure Statement}

No conflict of interests exists.

\section{References}

1 Ricard D, Idbaih A, Ducray F, Lahutte M, Hoang-Xuan K, Delattre JY: Primary brain tumours in adults. Lancet 2012;379:1984-1996.

-2 Gabayan AJ, Green SB, Sanan A, Jenrette J, Schultz C, Papagikos M, Tatter SP, Patel A, Amin P, Lustig R, Bastin KT, Watson G, Burri S, Stea B: GliaSite brachytherapy for treatment of recurrent malignant gliomas: a retrospective multi-institutional analysis. Neurosurgery 2006;58:701-709; discussion 701-709.

-3 Stupp R, Mason WP, van den Bent MJ, Weller M, Fisher B, Taphoorn MJ, Belanger K, Brandes AA, Marosi C, Bogdahn U, Curschmann J, Janzer RC, Ludwin SK, Gorlia T, Allgeier A, Lacombe D, Cairncross JG, Eisenhauer E, Mirimanoff RO, European Organisation for R, Treatment of Cancer Brain T, Radiotherapy G, National Cancer Institute of Canada Clinical Trials G: Radiotherapy plus concomitant and adjuvant temozolomide for glioblastoma. N Engl J Med 2005;352:987-996.

4 Orom UA, Shiekhattar R: Long noncoding RNAs usher in a new era in the biology of enhancers. Cell 2013;154:1190-1193.

5 Wapinski O, Chang HY: Long noncoding RNAs and human disease. Trends Cell Biol 2011;21:354-361.

6 Zhang JX, Han L, Bao ZS, Wang YY, Chen LY, Yan W, Yu SZ, Pu PY, Liu N, You YP, Jiang T, Kang CS, Chinese Glioma Cooperative G: HOTAIR, a cell cycle-associated long noncoding RNA and a strong predictor of survival, is preferentially expressed in classical and mesenchymal glioma. Neuro Oncol 2013;15:15951603.

7 Li Z, Xu C, Ding B, Gao M, Wei X, Ji N: Long non-coding RNA MALAT1 promotes proliferation and suppresses apoptosis of glioma cells through derepressing Rap1B by sponging miR-101. J Neurooncol 2017;134:19-28.

$>8$ Li H, Yuan X, Yan D, Li D, Guan F, Dong Y, Wang H, Liu X, Yang B: Long Non-Coding RNA MALAT1 Decreases the Sensitivity of Resistant Glioblastoma Cell Lines to Temozolomide. Cell Physiol Biochem 2017;42:11921201.

-9 Yu M, Ohira M, Li Y, Niizuma H, Oo ML, Zhu Y, Ozaki T, Isogai E, Nakamura Y, Koda T, Oba S, Yu B, Nakagawara A: High expression of ncRAN, a novel non-coding RNA mapped to chromosome 17q25.1, is associated with poor prognosis in neuroblastoma. Int J Oncol 2009;34:931-938.

10 Zhu Y, Yu M, Li Z, Kong C, Bi J, Li J, Gao Z, Li Z: ncRAN, a newly identified long noncoding RNA, enhances human bladder tumor growth, invasion, and survival. Urology 2011;77:510 e511-515. 


\section{Cellular Physiology Cell Physiol Biochem 2018;45:1975-1985

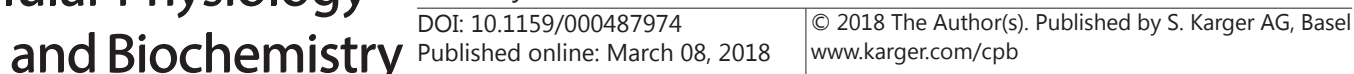

Lu et al.: SNHG16 Functions as an Oncogene in Glioma

11 Qi P, Xu MD, Ni SJ, Shen XH, Wei P, Huang D, Tan C, Sheng WQ Zhou XY, Du X: Down-regulation of ncRAN, a long non-coding RNA, contributes to colorectal cancer cell migration and invasion and predicts poor overall survival for colorectal cancer patients. Mol Carcinog 2015;54:742-750.

12 Cory S, Roberts AW, Colman PM, Adams JM: Targeting BCL-2-like Proteins to Kill Cancer Cells. Trends Cancer 2016;2:443-460.

13 Danielsen SA, Eide PW, Nesbakken A, Guren T, Leithe E, Lothe RA: Portrait of the PI3K/AKT pathway in colorectal cancer. Biochim Biophys Acta 2015;1855:104-121.

14 Guil S, Esteller M: RNA-RNA interactions in gene regulation: the coding and noncoding players. Trends Biochem Sci 2015;40:248-256.

15 Christensen LL, True K, Hamilton MP, Nielsen MM, Damas ND, Damgaard CK, Ongen H, Dermitzakis E, Bramsen JB, Pedersen JS, Lund AH, Vang S, Stribolt K, Madsen MR, Laurberg S, McGuire SE, Orntoft TF, Andersen CL: SNHG16 is regulated by the Wnt pathway in colorectal cancer and affects genes involved in lipid metabolism. Mol Oncol 2016;10:1266-1282.

16 Han W, Du X, Wang J, Sun L, Li Y: SNHG16 indicates a poor prognosis and affects cell proliferation, migration and invasion in non-small cell lung cancer. Exp Cell Res 2017

17 Eisele G, Weller M: Targeting apoptosis pathways in glioblastoma. Cancer Lett 2013;332:335-345.

18 Paw I, Carpenter RC, Watabe K, Debinski W, Lo HW: Mechanisms regulating glioma invasion. Cancer Lett 2015;362:1-7.

19 Dimitrova V, Arcaro A: Targeting the PI3K/AKT/mTOR signaling pathway in medulloblastoma. Curr Mol Med 2015;15:82-93.

20 Scoumanne A, Zhang J, Chen X: PRMT5 is required for cell-cycle progression and p53 tumor suppressor function. Nucleic Acids Res 2009;37:4965-4976.

-21 Yan F, Alinari L, Lustberg ME, Martin LK, Cordero-Nieves HM, Banasavadi-Siddegowda Y, Virk S, BarnholtzSloan J, Bell EH, Wojton J, Jacob NK, Chakravarti A, Nowicki MO, Wu X, Lapalombella R, Datta J, Yu B, Gordon K, Haseley A, Patton JT, Smith PL, Ryu J, Zhang X, Mo X, Marcucci G, Nuovo G, Kwon CH, Byrd JC, Chiocca EA, Li C, Sif S, Jacob S, Lawler S, Kaur B, Baiocchi RA: Genetic validation of the protein arginine methyltransferase PRMT5 as a candidate therapeutic target in glioblastoma. Cancer Res 2014;74:17521765.

22 Banasavadi-Siddegowda YK, Russell L, Frair E, Karkhanis VA, Relation T, Yoo JY, Zhang J, Sif S, Imitola J, Baiocchi R, Kaur B: PRMT5-PTEN molecular pathway regulates senescence and self-renewal of primary glioblastoma neurosphere cells. Oncogene 2017;36:263-274.

23 Han X, Li R, Zhang W, Yang X, Wheeler CG, Friedman GK, Province P, Ding Q, You Z, Fathallah-Shaykh HM, Gillespie GY, Zhao X, King PH, Nabors LB: Expression of PRMT5 correlates with malignant grade in gliomas and plays a pivotal role in tumor growth in vitro. J Neurooncol 2014;118:61-72. 